\title{
Reflexividad y narratividad en Ricardo Piglia: de Macedonio a Fitzgerald
}

\author{
Andrea Torres Perdigón \\ UNIVERSITE PARIS SORBONNE·Andreatorrespa@gmail.com
}

Prepara su tesis doctoral en literatura hispanoamericana en la Universidad de la Sorbona. Su investigación se centra en las nociones de metaficción y experiencia en Ricardo Piglia, Juan José Saer y Roberto Bolaño. Ha publicado varios artículos en revistas académicas de Europa y América Latina como Amerika: Mémoires identités, territoires; Perífrasis, Revista de Teoría, Literatura y Crítica; Les Ateliers du SAL y Revista Letral.

RECIBIDO: 24 DE ENERO DE 2013

ACEPTADO: 19 DE FEBRERO DE 2013

Resumen: Este artículo analiza algunos rasgos de la narrativa reciente del argentino Ricardo Piglia con relación a las poéticas de Macedonio Fernández y del estadounidense Francis Scott Fitzgerald. La primera parte trabaja la relación conflictiva de Piglia con el llamado posmodernismo; la segunda parte explica el vínculo entre Piglia y Macedonio, a través de la poética de la autonomía de lo literario; la tercera parte aborda la relación con Fitzgerald, por medio de un análisis de sus respectivas búsquedas de formas narrativas, para concluir en una cuarta parte ciertos cambios dentro de la propia obra novelesca de Piglia.

Palabras Clave: Piglia, Fitzgerald, Macedonio, posmodernismo, narratividad, metaficción.
Abstract: This article analyses some of the traits of Ricardo Piglia's recent narrative fiction by means of its relation to Macedonio Fernández and the American author Francis Scott Fitzgerald. The first part develops Piglia's conflictual approach to the postmodern. The second part explains the bond between Piglia and Macedonio by showing how their poetics defend the idea of literature's autonomy. The third part examines his relation to the oeuvre of Fitzgerald by analyzing their respective ways of conceiving narrative forms. The article concludes with a reading of some transformations within Piglia's novelistic works.

Key Words: Piglia, Fitzgerald, Macedonio, postmodernism, narrativity, metafiction. 
De todos modos, no me interesa la identificación con la vanguardia,jamás me definí desde ese lugar, aunque quizás en estos tiempos tan conservadores vendría bien reivindicar el espíritu de la vanguardia. Prefiero hablar de experimentación, que para mí es la marca de la gran literatura

RiCARDO PigLIA (2001[1985]]: 99)

A veces pienso que en esta época el mejor modo de ser un escritor experimental es escribir novelas tradicionales

RiCARDO Piglia (ENTREVISTA, 2010)

\section{Introducción: el lugar de Piglia en la narrativa contemporánea}

La obra narrativa y ensayística del escritor argentino Ricardo Piglia (Adrogué 1940) es hoy en día una de las más reconocidas y estudiadas de la literatura hispanoamericana contemporánea. Se le han dedicado, especialmente desde la década de los noventa, varios volúmenes críticos $^{1}$ y también se han publicado numerosos artículos en el ámbito hispanoamericano, estadounidense y europeo. Además se le han otorgado diversos premios, entre ellos el polémico² Premio Planeta Argentina en 1997, y en 2011 el Premio Internacional de Novela Rómulo Gallegos y el Premio Internacional de Novela Dashiell Hammett $^{3}$. La consolidación de su proyecto literario - llevada a cabo durante varias décadas - nos permite estudiar ciertos aspectos de la narrativa contemporánea escrita en español y en particular de la novela. El hecho de que hoy siga siendo un autor reconocido, y de que ese reconocimiento se haya consolidado en Hispanoamérica al menos desde la publicación de Respiración artificial en 1980 nos habla de ciertos aspectos de su literatura que han sido valorados por parte de la crítica desde aquel entonces. Estos aspectos tienen

${ }^{1}$ Solo por dar algunos ejemplos podemos mencionar los estudios de Nicolás Bratosevich (1997), Jorge Fornet (2000, 2005), Maria Antonieta Pereira (2001), Edgardo H. Berg (2003), E. Balder, Adriana Rodríguez Pérsico (2004), Rose Corral Ed. (2007), Jorge Carrión (2008).

${ }^{2}$ A propósito de esta polémica ver, por ejemplo, Susana, Reinoso. "Intelectuales respaldan a Piglia" (2013) o Estrada, Socorro. "Condenan a Ricardo Piglia y a la editorial por el premio Planeta" (2013).

${ }^{3}$ Aunque, como lo diría el mismo Piglia, "[...] los premios, obviamente, son una manifestación pura de la lógica del mercado, realizan directamente lo que el mercado insinúa, establecen una jerarquía fija, deciden que un libro es mejor que otro, y en ese sentido son la antítesis de la literatura [...]" (2001: 181). 
que ver principalmente con el debate sobre la posmodernidad, sobre las relaciones entre la historia, la política y la ficción o sobre las filiaciones entre tradiciones literarias ${ }^{4}$.

Ahora bien, al margen de estos aspectos, las diferencias entre las cuatro novelas de Piglia dan cuenta de un proceso de búsqueda de formas narrativas que llega hasta la publicación de Blanco nocturno en 2010. Esas diferencias nos permiten proponer un acercamiento a su obra a partir de su más reciente novela para pensar ciertas relaciones de continuidad o de ruptura con otras poéticas anteriores a él y con su propia trayectoria por el género de la novela.

La obra de Piglia se compone principalmente ${ }^{5}$ de cuatro novelas, dos libros de cuentos, uno de nouvelles, uno de entrevistas, y otros dos que alternan entre el género del ensayo y el del cuento. En particular, sus primeras dos novelas Respiración artificial(1980) y La ciudad ausente (1992) le otorgaron un importante reconocimiento como narrador, mientras que el libro de entrevistas Crítica y ficción (de 1986, pero reeditado con nuevos textos en 2001) y sus dos libros híbridos Formas breves (1999) y El último lector (2005) contribuyeron a la imagen de escritor-crítico que actualmente es casi una marca distintiva de su producción. Sus dos últimas novelas, Plata quemada (1997) y Blanco nocturno (2010), cumplieron el papel de fortalecer su lugar como escritor; Plata quemada fue un gran éxito editorial y sobre ella se hizo una película homónima dirigida por Marcelo Piñeyro en 2000, y con Blanco nocturno ganó en 2011 dos de los premios mencionados anteriormente.

Independientemente de que se quiera ver la misma calidad literaria en estas últimas novelas que en las dos primeras, lo que nos interesa es la transformación de la escritura de Piglia, especialmente en el caso de su novela más reciente, Blanco nocturno. El objetivo es entonces observar qué rasgos de esta última novela están vinculados con la producción anterior de Piglia, y cuál es la relación con proyectos literarios de la primera mitad del siglo XX, en especial con algunos textos de Macedonio Fernández (Buenos Aires 1874-1952) y de Francis Scott Fitzgerald (St. Paul Minnesota 1896 - Los Angeles 1940).

\section{Piglia y la poética del posmodernismo}

Para comenzar, y puede que esta sea una característica común a varias novelas contemporáneas, en Piglia hay un agotamiento y una suerte de rechazo a lo que se identificó

\footnotetext{
${ }^{4}$ Ver, por ejemplo, la lectura de Sandra Garabano en Reescribiendo la nación, la narrativa de Ricardo Piglia: "La obra de Piglia [...] coincide con un verdadero auge de las teorías recientes sobre el posestructuralismo y la posmodernidad" (2003: 21) y más adelante "La obra de Piglia interviene en este debate con preguntas sobre la identidad, la voz y, sobre todo, la idea de representación, no sólo estética sino política. Además, sus escritos reconstruyen varias de las oposiciones que han caracterizado a la literatura argentina e investigan sobre las paradojas creadas por la historiografía al intentar representar la historia" (2003: 22).

${ }^{5}$ Decimos principalmente porque hay una serie de artículos críticos y de entrevistas que no han sido compilados en libros, pero que hacen parte de la producción intelectual del autor.
} 
como posmodernismo durante las últimas décadas del siglo XX. Esta actitud puede verse, por una parte, en los textos de orden ensayístico, y por otra, en la forma narrativa de algunas de sus novelas, como lo mostraremos a continuación.

\subsection{La incomodidad de lo posmoderno}

En el primer caso, Piglia manifiesta una molestia respecto a la actitud de cierto tipo de crítica que le atribuye a la literatura el papel de un documento más, dentro del conjunto de documentos culturales o sociales que circulan en el mundo contemporáneo. Así, el texto literario termina leyéndose como un signo de elementos sociales que habría que entrar a descifrar. En una entrevista realizada en 1998, el autor argentino afirma que

[...] muchas tácticas críticas tienden a hacer eso. Tienden, desde posiciones que se suponen progresistas, a sacar la literatura del juego y a convertirla en un síntoma más de una serie de documentos sociales que circulan con el mismo estatuto que la literatura. La crítica tiende a ver a la literatura como un síntoma, como un síntoma de otra cosa. La literatura no es síntoma de otra cosa (2001: 174).

El primer punto que incomoda a Piglia tiene que ver entonces con la uniformización del tratamiento del texto literario respecto a múltiples manifestaciones sociales o culturales, uniformización que se produce en el contexto de los estudios sobre lo posmoderno. Hay en estos una suerte de extrapolación del concepto foucaultiano de discurso, en la cual todas las manifestaciones culturales son estudiadas de la misma manera como documentos que atestiguan la presencia y el funcionamiento de ciertas estructuras sociales o de paradigmas discursivos. Desde luego, esta lectura de la literatura como síntoma o como documento puede ser fructífera, pero Piglia aboga por la defensa de un espacio para lo específicamente literario; una lectura de la literatura por fuera de su carácter de documento cultural y de su eventual interés antropológico en este sentido. Para él, la crítica debe dedicarse a ese lugar literario y no al documento como tal.

Un segundo punto que refleja el cansancio de la tendencia académica dominante de finales del siglo XX es que Piglia expresa abiertamente su desacuerdo con uno de los términos clave para entender los procedimientos literarios llamados posmodernos. se trata de la metaficción. Este término está ligado al posmodernismo entendido como movimiento cultural. Para Patricia Waugh, por ejemplo, "Metafiction is a mode of writing within a broader cultural movement often referred to as post-modernism" (1984: 21) y "Although metafiction is just one form of post-modernism, nearly all contemporary experimental writing displays some explicitly metafictional strategies" (1984: 22). Para Waugh, si bien la metaficción no era considerada la única característica de la escritura posmoderna, sí definía el carácter experimental de una novela, al menos en la década de los ochenta. Respecto al 
término de metaficción y su uso a propósito de la narrativa, Piglia acota que le resulta superfluo, ya que le parece "[...] que la ficción es siempre metaficción" (2001: 188). En la misma entrevista de 1998 citada anteriormente, Piglia afirma:

A mí ese debate entre metaficción y narración directa no me interesa, ni entro en eso, porque creo que uno puede encontrar metaficción en los narradores que parecen más ingenuos y más en ellos que en otros, diría yo. [...] Pero la metacrítica, o sea, el uso de la crítica en un espacio distinto, que sería el modo en el que yo entiendo la cuestión, sí me interesa mucho [...] (2001: 189).

En la concepción pigliana de la literatura, la ficción puede ser entonces reflexiva por principio; así, de manera más o menos explícita la metaficción haría parte del ejercicio literario, y por ende le resulta irrelevante tratar de forzar el uso de un concepto crítico como el de metaficción como rasgo distintivo de la literatura posmoderna. Ahora bien, lo que rescata es el ejercicio de una crítica dentro de la ficción; es decir, no la reflexividad que acompañaría en principio cualquier gesto literario, sino la reflexividad en términos de un pensamiento crítico de la idea de la literatura como parte de los materiales a partir de los cuales se pueden construir ficciones.

Ahora bien, hay un tercer punto del posmodernismo a propósito del cual Piglia manifiesta cierto desacuerdo en sus textos críticos. Este punto tiene que ver con un conjunto de conceptos que privilegian lo impreciso, lo disperso, lo vago, lo paradójico y la pérdida de límites entre discursos y nociones. En términos de Linda Hutcheon,

The multiple, the heterogeneous, the different: this is the pluralizing rhetoric of postmodernism that rejects both the abstract category of single otherness created by "coercive separation and unequal privileges" (Said 1985,43) as well as by the more concrete relegation of the other to the role of "object for enthusiastic information-retrieval" (Spivak 1985, 245). The language of margins and borders marks a position of paradox: both inside and outside (1988: 66).

Se trata del posmodernismo ligado a nociones como lo múltiple o lo heterogéneo, entre otros. Ahora bien, para Hutcheon todo ese discurso de los márgenes y de los bordes está marcado por "una posición paradójica", característica que le parece fundamental al hablar de una poética común del fenómeno cultural del posmodernismo:

We have seen that what both its supporters and its detractors seem to want to call 'postmodernism' in art today - be it in video, dance, literature, painting, music, architecture, or any other form - seems to be art marked paradoxically by both history and an internalized, self-reflexive investigation of the nature, the limits, and the possibilities of the discourse of art (1988: 22). 
Para Hutcheon, el rasgo común a las múltiples descripciones y críticas del posmodernismo - en cuanto al arte se refiere - es que la historia y la reflexividad sobre el arte mismo aparecen simultáneamente y, sobre todo, de manera paradójica, es decir, que su encuentro no se resuelve: "There is no dialectic in the postmodern: the self-reflexive remains distinct from its traditionally accepted contrary -the historico-political context in which it is embedded" (Hutcheon 1988: x). Ya sea hablando de la historia y la reflexividad en el arte, o de lo heterogéneo, diferente y múltiple, lo que más destaca Hutcheon es la idea de que todos esos componentes se mantienen como una tensión, como una situación paradójica, sin solución.

Ahora bien, esta valoración de la irresolución o la indefinición como rasgo determinante de lo posmoderno, es similar a otro aspecto que Peter Hallward critica al hablar de un paradigma conceptualmente cercano al del posmodernismo: el de los estudios poscoloniales. Para Hallward,

The signature postcolonial concepts - the hybrid, the interstitial, the intercultural, the in-between, the indeterminate, the counter-hegemonic, the contingent, and so on - are so many attempts to evoke that which no concept can 'capture'. Spivak's aversion to 'general intelligibility, general or universal equivalences' is typical of the field as a whole $(2001, \mathrm{xi})$.

Consideramos que el recurso a conceptos que buscan nombrar entidades inaprensibles o ininteligibles -o por lo menos no generalizables - es común al campo de los estudios coloniales y al del posmodernismo. En la visión de Hutcheon de lo posmoderno, por ejemplo, el rasgo recurrente es la paradoja. hay elementos que se mantienen en tensión, pero que no se resuelven de ninguna manera, quedan permanentemente en el ámbito de lo indefinido. Sin embargo, además de lo paradójico, conceptos como lo híbrido, lo indeterminado, lo contra-hegemónico, pueden pensarse también para hablar de literatura posmoderna. Ahora bien, volviendo a Piglia, el punto particular que él señala es el de la pérdida de límites, en especial en lo que concierne la ficción y la realidad. Al respecto, Piglia nos dice que

Después está esta cuestión que aparece tan a menudo en las discusiones en las que estamos los escritores, los críticos o los historiadores, que tiene que ver con que todo es ficción, que es como una situación que está muy presente en el discurso histórico y en el debate cultural. Me parece que ahí se produce una extrapolación de algo que uno podría localizar más precisamente y decir que es en la cultura de masas donde la distinción entre ficción y verdad se ha perdido. Y que muchos de los filósofos 'posmodernos' - entre comillas- trasladan lo que es real en la cultura de masas al conjunto de las prácticas (2001: 211). 
Como puede observarse hay una cierta preocupación en Piglia por el término verdad y por el auge del relativismo cultural en las ciencias humanas, y no solo en lo que se refiere a la literatura o los estudios literarios. Ese relativismo es visto por Piglia como parte del discurso de la cultura de masas, mas no como un elemento epistemológico que pueda operar para explicar prácticas sociales. De alguna forma Piglia indica que no se trata de decir que no hay diferencia entre ficción y realidad, y que esa postura no es productiva ni para el estudio de la literatura, ni probablemente para el del ámbito social. Piglia insiste en que esa extrapolación:

nos lleva, a menudo, hoy, a una concepción de la verdad que no es pertinente porque pertenece a ese ámbito preciso y no a todas las prácticas de la sociedad. Los filósofos 'posmodernos' son filósofos de la cultura de masas y ven el mundo bajo la forma de la cultura de masas (2001: 212).

Como se ha reiterado, la posición de Piglia respecto al movimiento cultural posmoderno es más bien cautelosa e incluso desconfiada, particularmente en Crítica y ficción, donde el tema todavía hacía parte de los debates académicos y culturales. Dicho esto y después de haber enunciado su mirada crítica frente a la poética posmodernista de los años ochenta, ¿qué rasgos determinan entonces la narrativa de Piglia?, ¿en qué consiste su alejamiento de estas posturas en sus novelas?

\subsection{La búsqueda de otra forma de narratividad}

El cansancio de lo que se identifica como posmoderno se ve también en las formas narrativas que Piglia adopta, en particular en las últimas dos novelas, Plata quemada y Blanco nocturno. Estas dos novelas se alejan de elementos formales precisos como el fragmentarismo - entendido como presentación discontinua del relato- y la reflexión metaficcional como eje formal de la novela.

Como se ha indicado en The Cambridge Introduction to Postmodern Fiction, la "discontinuous and fragmented narrative (considered one of the novel's most postmodern elements)" (Nicol 2009: 129) se convirtió en uno de los rasgos dominantes de la ficción posmoderna. $\mathrm{O}$, como nos indica Hutcheon, explicando la perspectiva de continuidad entre el modernismo y el posmodernismo: "On a formal level, modernism and postmodernism are said to share self-reflexivity (Fokkema 1984, 17), fragmentation (Newman 1985, 113), and a concern for history (literary and social) (Thiher 1984, 216 19)" (1988: 51). En el caso de las últimas dos novelas de Piglia el fragmentarismo como rasgo formal de la narrativa está casi ausente; y la reflexividad, si bien continúa ocupando 
un lugar destacado, no es un rasgo formal dominante ${ }^{6}$, como tal vez sí lo era en Respiración artificial y La ciudad ausente.

A partir de lo anterior, nos interesa postular que la marginación de estos dos rasgos formales - el fragmentarismo y la reflexividad-implican la adopción de otro tipo de estructuras que parecen recuperar elementos temáticos de la vanguardia de Macedonio Fernández, por un lado, y elementos formales de la narrativa del estadounidense Francis Scott Fitzgerald, como veremos en las páginas que siguen. En ambos casos, se puede rastrear un vínculo entre los proyectos literarios de estos dos autores de comienzos del siglo XX y las formas dominantes en la novela más reciente de Ricardo Piglia.

\section{Macedonio: la autonomía de la literatura como tema}

La presencia de Macedonio Fernández en la obra de Piglia es explícita tanto en su ficción como en sus textos de orden ensayístico. Ya en La ciudad ausente, Macedonio aparece como personaje dentro de la novela, mientras que en Formas breves es el tema de uno de los relatos-ensayo. Ahora bien, la figura de Macedonio representa ante todo una poética en la cual la literatura es un arte de lo posible, radicalmente autónomo e independiente de la representación de cualquier tipo de realidad que no sea la suya propia. En uno de los prólogos al Museo de la Novela de la Eterna, Macedonio explica parte de esta poética:

Lo que no quiero y veinte veces he acudido a evitarlo en mis páginas, es que el personaje parezca vivir, y esto ocurre cada vez que en el ánimo del lector hay alucinación de realidad del suceso: la verdad de la vida, la copia de la vida, es mi abominación, y ciertamente, $\mathrm{c}^{\text {no }}$ es lo genuino del fracaso de arte, la mayor, quizá la única frustración, abortación, que un personaje parezca vivir? Yo consiento que ellos quieran vivir, en el sentido de que intenten y codicien la vida, pero no que parezcan vivir, en el sentido de que los sucesos parezcan reales: abomino de todo realismo (1996: 40).

El principal gesto de vanguardia en Macedonio es su insistente defensa de la autonomía de la literatura, por encima de cualquier tipo de representación de lo real. Piglia, sin adoptar la forma ni la vía estructural de la novela de Macedonio, se apropia de esta visión de la literatura para transformarla en un tema para sus ficciones. De ahí que una buena parte de sus reflexiones sobre la literatura aludan con frecuencia a esta como una articulación permanente entre el pensamiento y la ficción; como un ámbito de lo posible. En Formas breves Piglia le atribuye a la poética de Macedonio esta visión de la literatura:

'Pero hay otra cuestión', dice Renzi. 'CCuál es el problema mayor del arte de Macedonio? Las relaciones del pensamiento con la literatura.' El pensar, diría

${ }^{6}$ No nos ocuparemos aquí del asunto del interés por la historia, aunque tiene un papel más o menos destacado, en particular en Blanco nocturno. 


\begin{abstract}
Macedonio, es algo que se puede narrar como se narra un viaje o una historia de amor, pero no del mismo modo. Le parece posible que en una novela puedan expresarse pensamientos tan difíciles y de forma tan abstracta como en una obra filosófica, pero a condición de que parezcan falsos. 'Esa ilusión de falsedad', dijo Renzi, 'es la literatura misma' (2000: 28).
\end{abstract}

Como se observa en esta cita, la figura de Macedonio le sirve a Piglia para reflexionar acerca de esta idea de literatura - que en el fondo describe también su propia poética- y al tiempo para crear su relato-ensayo 'Notas sobre Macedonio en un diario'. Esta autonomía de la ficción aparece la mayoría de veces como un tema de digresión, como en el ejemplo anterior, y como un motivo narrativo - en especial a través de la máquina de La ciudad ausente- Pero, en un sentido estructural, también opera a través de la experimentación formal en Respiración artificial y La ciudad ausente, por medio de la intercalación de múltiples historias y de cierta indeterminación de los narradores. Consideramos entonces que estos rasgos experimentales de sus primeras dos novelas se originan en parte en su lectura y su interés por trabajar y reivindicar la idea de literatura de Macedonio. En esta medida, lo experimental en las primeras dos novelas de Piglia puede estar más cerca de la vanguardia que de lo posmoderno; la multiplicidad de historias y la indeterminación de los narradores pueden responder entonces más al ideal de la autonomía formal del artificio literario, que al reflejo de un mundo disperso y fragmentado posterior a la desconfianza de los grandes metarrelatos, en el sentido de Jean-François Lyotard ${ }^{7}$.

Sin embargo, en Plata quemada y Blanco nocturno, la poética macedoniana como experimentación formal se reduce significativamente con respecto a las dos novelas anteriores. De hecho, en Blanco nocturno la presencia de esta vanguardia macedoniana se reduce a ciertos personajes que oscilan entre la cordura y la demencia, como Croce o Luca. En el caso de Luca, por ejemplo:

Lo acusaban de ser irreal, de no tener los pies en la tierra. Pero había estado pensando, lo imaginario no era lo irreal. Lo imaginario era lo posible, lo que todavía no es, y en esa proyección al futuro estaba, al mismo tiempo, lo que existe y lo que no existe (Piglia 2010: 232).

El personaje Luca reitera la función del artista-ingeniero que trabaja con lo irreal, lo imaginario y lo posible, función que poseen por ejemplo Russo o la máquina como metáfora de la escritura en La ciudad ausente, y que está ligada a la búsqueda de la autonomía de la literatura de Macedonio. Pero, aparte de estos rasgos de unos cuantos personajes, la idea vanguardista macedoniana en términos de experimentación narrativa casi desaparece y queda operando únicamente como tema dentro de la narración.

7 "En simplifiant à l'extrême, on tient pour 'postmoderne' l'incrédulité à l'égard des métarécits" (1979: 7). 
Ahora bien, las reflexiones sobre la literatura siguen haciendo parte de la narración pigliana casi en todos sus textos novelísticos —salvo en Plata quemada donde probablemente el gesto aparece de manera explícita solo en el Epílogo-. No obstante, en Blanco nocturno estas reflexiones son marginales y menos recurrentes que en las novelas de 1980 y 1992. De hecho, el argumento de esta novela está vinculado explícitamente a un contexto histórico y al ámbito rural argentino de manera transparente. En una nota al pie atribuida al narrador, se afirma expresamente que:

Diez años después de los hechos registrados en esta crónica, en las vísperas de la guerra de las Malvinas, Renzi leyó en The Guardian que los soldados ingleses estaban provistos de anteojos infrarrojos que les permitían ver en la oscuridad y disparar sobre un blanco nocturno y se dio cuenta de que la guerra estaba perdida antes de empezar [...] (2010: 149).

De esta manera se sabe que la novela transcurre en el año 1972: el argumento se vincula así a un contexto histórico preciso y al ámbito rural de la Argentina de manera transparente y clara. En este sentido, Blanco nocturno está cerca de Plata quemada, en la medida en que el mundo referencial y la historia tienen un papel destacado y más explícito que en La ciudad ausente. En esta medida, el papel de las digresiones sobre la literatura se limita a ciertos momentos de la segunda parte de la novela y a algunas notas al pie atribuidas a Renzi, como la que presentamos a continuación:

'El capítulo 10 de la novela - The Law-Writer- está centrado en el copista Nemo (Nadie). Publicado en Nueva York en la revista Harper en abril de 1853, fue seguramente leído por Melville, que escribió Bartleby en noviembre de ese año. La novela de Dickens, que narra un juicio interminable con su mundo de tribunales y de jueces, fue un libro muy admirado por Kafka' (nota de Renzi) (2010: 271).

Como se aprecia, las digresiones críticas se conservan, aunque de manera marginal respecto a la historia familiar narrada en la novela. Además, las notas atribuidas a Renzi permiten que haya cierta verosimilitud y justificación de su aparición en calidad de notas al pie, preocupación que no es tan visible en la experimentación de La ciudad ausente.

Ahora bien, hay otro aspecto reflexivo de esta novela que corresponde a las afirmaciones sobre el género policial. Aunque no tienen ya relación con la poética de Macedonio, hacen parte del corpus de reflexiones que la novela enuncia. A través de la voz del detective Croce, se afirma respecto al género policial que "No es cierto que se pueda restablecer el orden, no es cierto que el crimen siempre se resuelve... No hay ninguna

8 Cabe anotar que en Plata quemada hay una frase similar, que identifica la novela que se está leyendo con una crónica también. Se trata de un motivo recurrente: "La gran odisea que ya dura cuatro horas en el momento de escribir esta crónica comenzó aproximadamente las 22 horas de ayer y [..." " (2000: 155). 
lógica" (2010: 283). Parte de la estructura de esta novela reside precisamente en esto: usar el género policial pero sin responder a las expectativas de la lectura; sin resolver el crimen. En este sentido la reflexividad sigue operando, aunque dentro de una literatura más lineal y secuencial, y por lo tanto más tradicional.

\section{Fitzgerald: formas de narratividad y reflexividad}

Uno de los autores que Piglia reivindica por fuera de la tradición argentina es el estadounidense Francis Scott Fitzgerald. Aun cuando se trata de un escritor de otra tradición y otra lengua, su estética y ciertos elementos de sus novelas pueden darnos algunas luces acerca del proyecto literario que realiza Piglia, en particular sabiendo que la relación con la literatura norteamericana es relevante en su proyecto estético ${ }^{9}$.

Ahora bien, la relación de Piglia con la obra de Fitzgerald es distinta de la que se establece con Macedonio. Si frente a este último hay una fascinación por la potencia y la capacidad sugestiva de su proyecto como máxima autonomía del artificio literario, con Fitzgerald el vínculo pasa más por la búsqueda de cierto tipo de códigos narrativos. Además de aspectos temáticos como la aparición de personajes con problemas de salud mental o la narración de la pérdida, la escritura de Piglia se vincula formalmente con la de Fitzgerald básicamente a través de dos puntos: en primer lugar, el mantenimiento de un nivel de la historia que se puede reconstruir como una secuencia de eventos, y que en esa medida es lineal - a pesar de las analepsis o prolepsis en el nivel del relato tanto en los textos de Fitzgerald como en los de Piglia - ; se trata de una forma de narración que, al articular episodios secuencialmente en el nivel de la historia, resulta diferente de una narrativa realmente 'fragmentaria' - tan presente en la llamada posmodernidad-. En segundo lugar, una cierta forma de integrar y de presentar digresiones o reflexiones que están por fuera de la línea de la trama es común a Fitzgerald y a Piglia; la digresión se integra en el nivel del relato que constituye la novela. En esta medida, tanto las digresiones en Fitzgerald sobre diversos aspectos de la experiencia, como las reflexiones sobre la literatura en Piglia, tienen un espacio concomitante respecto a la historia que se está contando, sin que se constituyan en el núcleo de la narración.

La obra de Francis Scott Fitzgerald ha sido ampliamente estudiada y comentada, sobre todo desde dos perspectivas: una biográfica y otra sociológica. La primera se explica por la fascinación que ejerció y que ejerce aún su figura y la de su esposa Zelda. Como lo ha señalado ya Curnutt en The Cambridge Introduction to F. Scott Fitzgerald,

\footnotetext{
${ }^{9}$ Como lo indica Adriana Rodríguez Pérsico en su artículo "Introducción a Ricardo Piglia" de 1990: "[...]Piglia se desliza por los meandros de la cultura argentina, se asoma a su historia, transita otras literaturas, demorándose con especial énfasis en la norteamericana [...]” (2000: 46).
} 
Biography is by far the most prolific branch of contemporary criticism, with upwards of a dozen fulllength volumes devoted to Scott's and Zelda's life together (not including various reminiscences by friends and acquaintances). Within this field one encounters the most contested issue concerning the couple -namely, the nature of their marriage and how it affected their work (2007: 123).

La otra vía crítica que ha dominado los estudios sobre su obra está marcada por intereses sociológicos respecto a la época posterior al fin de la Primera Guerra Mundial, y respecto a cómo sus obras hablan de varios aspectos de la sociedad norteamericana de los años veinte y treinta. Esta fue, sobre todo, la primera recepción de sus textos: "Although his métier was fiction, many reviewers were more interested in what he had to say about his generation's sociology" (Curnutt 2007: 112). Dejando de lado este aspecto del autor como voz o símbolo de una generación determinada, nos centraremos acá en los dos aspectos formales de su narrativa que - pensamos- están relacionados con la búsqueda estética más reciente de Ricardo Piglia: la secuencialidad en el nivel de la historia y la intercalación de la digresión y la narración.

Novelas como The Great Gatsby (1925) y Tender is the Night (1934) - siendo muy distintas una de la otra- exponen una articulación de múltiples elementos. Entre ellos, Curnutt trabaja en particular la presencia de movimientos literarios que se reconocen de diversas maneras y aparecen con distinta intensidad. Miraremos brevemente solo los tres primeros movimientos o modos de escritura que marcarían el desarrollo de estas novelas de Fitzgerald $-\mathrm{y}$ de su poética en general según Curnutt— para ver cuáles son los rasgos que podríamos vincular con Piglia.

\subsection{Modernismo, romanticismo y realismo}

En primer lugar está el carácter modernista —en el sentido que esta palabra tiene en el contexto anglosajón-, en cuanto a la actitud frente al lugar que ocupa el argumento dentro de las novelas. Como lo explica Curnutt,

From Joyce to Virginia Woolf and even Hemingway, plotting was deemed more appropriate for melodramatic potboilers than literature, whose proper concern was consciousness and character. Like these peers, Fitzgerald considered unity a matter of atmosphere, not story logic (2007: 46).

Como se observa, uno de los rasgos modernistas en Fitzgerald consiste en un principio singular de unidad de la novela: este no está dado solo por la presentación de un argumento o de una trama (plot), sino que se manifiesta a través de otros aspectos que el término atmósfera engloba, y que tendrían que ver más con el estilo y el tono general de la narración. En este punto, encontramos que en Blanco nocturno Piglia retoma la misma idea - tanto del modernismo en general, como de Fitzgerald — de una unidad para la novela que 
no pasa por la lógica de la historia; como lo hemos mencionado ya, el crimen no se resuelve nunca en este texto y, aun así, los episodios transcurren sin que la acción -aparentemente central - del crimen tenga que desarrollarse y llegar a un desenlace. La unidad de la novela está dada, como en el caso de Fitzgerald, por una secuencia más o menos lineal de episodios que conservan un mismo tono y que crean una misma atmósfera. En Piglia, el argumento como encadenamiento de acciones - aunque persiste en las dos últimas novelas con mayor insistencia que en las dos anteriores - mantiene ese lugar un poco secundario que posee también en Fitzgerald ${ }^{10}$. Lo indispensable y lo que da unidad a una novela no es tanto la historia que se está narrando, sino la búsqueda de un tono. En palabras del propio Piglia en una entrevista de 1985: "El problema para mí no es armar la trama, sino encontrar el tono de un relato. Narrar es narrar en un ritmo, en una respiración del lenguaje: cuando uno tiene esa música la anécdota funciona sola, se transforma, se ramifica" (2001: 99). Sin embargo hay que insistir en que la trayectoria de Piglia por la novela parece llevarlo en sus últimas obras cada vez más hacia la recuperación de la trama, aun cuando no se constituya en un elemento acabado y completamente definido. Este no era el caso de La ciudad ausente, donde la reconstrucción de una trama única resulta innecesaria para el desarrollo de la novela.

Al volver a Fitzgerald encontramos que, en segundo lugar y más que nada con relación a los temas, se ha estudiado el vínculo de su obra con el romanticismo: el interés por narrar la pérdida y la caída, así como la brecha infranqueable entre lo ideal y lo real son temas recurrentes tanto en Tender is the Night como en The Great Gatsby. Ahora bien, estamos de acuerdo con Curnutt cuando afirma que:

Fitzgerald's Romanticism was atypical of his time, for such leading literary peers as T.S. Eliot and Ezra Pound had made the mode unfashionable by attacking it as excessively emotional and escapist. The modernist aesthetic they defined insisted on depersonalizing art with radically experimental styles, an approach that might not seem compatible with such an insistently autobiographical writer as Fitzgerald (2007: 100).

En efecto, al menos en las dos novelas que hemos mencionado la escritura de Fitzgerald, si bien margina un poco la importancia del argumento, no corresponde con la búsqueda formal y experimental de otros modernistas en lengua inglesa, como tampoco lo hacen sus temas de tendencia romántica. Las estructuras narrativas de Fitzgerald no son

\footnotetext{
${ }^{10}$ Puede pensarse en la importancia de la descripción inicial en The Great Gatsby, donde la lentitud de la acción funciona para crear cierto misterio en torno a Gatsby. Después de las largas descripciones, la acción se desarrolla de manera más acelerada hacia el final. También en Tender is the Night, es más determinante el proceso de caída de Dick y Nicole, que los eventos particulares que se narran.
} 
experimentales en el sentido de The Waves de Virginia Woolf o de Ulysses de James Joyce. Según Curnutt, al contrario del ideal modernista de despersonalizar el artificio literario, Fitzgerald construye sus ficciones siempre a partir de la experiencia. Como lo expone el mismo Fitzgerald en "One hundred false starts" (1933):

Mostly, we authors must repeat ourselves - that's the truth. We have two or three great and moving experiences in our lives - experiences so great and so moving that it doesn't seem at the time that anyone else has been so caught up and pounded and dazzled and astonished and beaten and broken and rescued and illuminated and rewarded and humbled in just that way ever before. Then we learn our trade, well or less well, and we tell our two or three stories —each time in a new disguise - maybe ten times, maybe a hundred, as long as people will listen (Fitzgerald 2005: 86).

Así las historias por contar sean pocas, la narración para Fitzgerald surge de un trabajo ficcional que parte siempre de la experiencia. Esto no quiere decir que su obra esté reducida a aspectos autobiográficos, sino que el material para desarrollar sus historias debe partir de la inmediatez de lo vivido. Para que el material pueda generar una historia vívida, Fitzgerald considera que la emoción debe haber sido experimentada y comprendida. De nuevo en sus palabras: "Whether it's something that happened twenty years ago or only yesterday, I must start out with an emotion -one that's close to me and that I can understand" (Fitzgerald 2005: 87).

Ahora bien, la presencia de esta narrativa de la propia experiencia nos lleva al tercer modo de escritura en Fitzgerald: el realismo. Según Curnutt,

Compared with his Romantic and modernist affinities, Fitzgerald's realist influences have received comparatively little attention. His fixation with class owes a debt to Henry James, Edith Wharton (1862-1937), and other late nineteenth-century writers concerned with how privilege and money shape moral character (2007: 103).

En este punto de contacto con el realismo se encuentra otro de los elementos que nos interesa, no tanto por la preocupación por el carácter moral - que puede estar también presente en Plata quemada, por ejemplo- sino por los códigos narrativos empleados para hablar de ese carácter. La escritura de Fitzgerald comparte ciertas características formales con literaturas consideradas realistas, y aquí el vínculo con Piglia vuelve a aparecer: además de la secuencialidad de los episodios en el nivel de la historia, el tipo de narrador que ambos autores utilizan los acerca a ciertas convenciones del realismo literario del siglo XIX.

\subsection{Narrador y focalización}


Los narradores de Blanco nocturno y de Tender is the Night poseen dos rasgos en común que tienen que ver con una forma particular: se trata de una forma que privilegia el impacto del estilo, por encima del de la estructura general de la novela y de su argumento. La primera de estas dos características es una focalización más o menos estable que tiende a ser neutra. Aunque Blanco nocturno tiene dos narradores - una voz extradiegética anónima y Sofía, uno de los personajes - el papel principal lo tiene la voz impersonal y extradiegética cuya focalización puede pasar perfectamente por tradicional, como se observa en el íncipit de la novela: "Tony Durán era un aventurero y un jugador profesional y vio la oportunidad de ganar la apuesta máxima cuando tropezó con las hermanas Belladona" (2010:13).

En términos de la focalización, los dos íncipit de Tender is the Night no difieren en gran medida: "On the pleasant shore of the French Riviera, about half way between Marseilles and the Italian border, stands a large, proud, rosecolored hotel. Deferential palms cool its flushed façade, and before it stretches a short dazzling beach". (Citado en Bruccoli y Parker Anderson (eds.), 2003: 200). En la edición de 1951 encontramos una perspectiva similar, aun cuando el inicio habla de Dick, el protagonista: "In the spring of 1917, when Doctor Richard Diver first arrived in Zürich, he was twenty-six years old, a fine age for a man, indeed the very acme of bachelorhood" (1953: 3). Como se puede observar en estas dos versiones ${ }^{11}$ de la novela de Fitzgerald la focalización tiende a ser neutra ya que la información que el narrador ofrece no está limitada a la perspectiva o al conocimiento de un personaje dado. Como lo aclara Burkhard Niederhoff "To tell a story from a character's point of view means to present the events as they are perceived, felt, interpreted and evaluated by her at a particular moment" (2009: 117). Como hemos visto, esto no es el caso en los dos comienzos de Fitzgerald y en el de Piglia.

Ahora bien, esta focalización neutra es relativamente estable durante el desarrollo de las dos novelas y no son comunes las focalizaciones internas ${ }^{12}$ en la voz de estos narradores extradiegéticos; es decir, el lugar en el cual se ubica la mirada de la narración es con frecuencia el mismo. Tanto en el caso de Piglia como en el de Fitzgerald no se trata en

${ }^{11}$ A propósito de las diferencias de estas dos ediciones ver la edición de Cambridge publicada en 2012: "Scribners attempts to make both versions available, but the reordered text does not gain acceptance and falls out of print during the 1960s. The 1934 text becomes the established version for teachers and critics" (West, 2012: xiii). Para complementar la información sobre las correcciones posteriores a 1934 y la edición póstuma de Cowley de 1951 ver también Bruccoli y Anderson (181-202) y la "Introduction" de Cowley (Cowley ix-xviii). Igualmente se puede consultar "The Text Itself" de Milton R. Stern : "In sum, it is totalitarian to declare as a matter of fact or a matter of settled opinion that one or the other version is the only version that should be considered" (1986: 25) y más adelante "The choice remains one of preference, not a choice between the 'real' book and something else" (1986:27).

${ }^{12}$ Cf. Gérard Genette (2007). Discours du récit. Paris: Editions du Seuil. 
general de narradores que adopten la perspectiva de alguno de los personajes - a pesar de que los capítulos de Tender is the Night estén marcados por episodios particulares que hablan de Rosemary, de Dick o de Nicole ${ }^{13}$ - En las dos versiones de Fitzgerald y en la novela de Piglia hay narradores que mantienen su relato con una focalización mínima; no proporcionan o restringen la información en función de la perspectiva específica de un personaje dado y tienden más bien a representar una perspectiva exterior, perspectiva que tiende a asociarse a una convenciones realistas.

En el caso de las novelas que hemos mencionado, los dos narradores —el de Piglia y el de Fitzgerald - permanecen entonces por fuera de las historias y no se guían por la perspectiva de ningún personaje específico, es decir que tienden a una focalización cero ${ }^{14}$. Por supuesto, esta característica se identifica con los códigos narrativos que se le atribuyen al realismo del siglo XIX y también al ilusionismo estético ${ }^{15}$ del relato tradicional, y se relaciona específicamente con la técnica de un narrador omnisciente. Respecto al tipo de narrador usado por el autor estadounidense se ha afirmado que:

Another misunderstood aspect of Fitzgerald's style is his preferred point of view for narrating his fiction. Whether writing in the first or the third person, he typically assumed an omniscient perspective that allowed him to 'get the verisimilitude of a first person narrative with a Godlike knowledge of all that happens to my characters" (A Life in Letters 410)" (A Life in Letters 410). (Curnutt, 2007: 109).

Dicho esto, la dominancia de este narrador extradiegético tradicional - tanto en las novelas más recientes de Piglia como en Fitzgerald- posee otra característica que, desde este punto de vista, hace parte de esa búsqueda de formas narrativas que anunciábamos al inicio. El narrador de Tender is the Night incurre con frecuencia en comentarios de este orden:

\footnotetext{
${ }^{13}$ Estos señalamientos aparecen explícitamente en la edición de Tender is the Night establecida por Malcolm Cowley en 1951 y no en la primera de 1934, en particular a través del título « Rosemary's Angle », del libro II.

${ }^{14}$ En el sentido en que Genette entendía este término en 1972 : "Nous rebaptiserons donc le premier type, celui que représente en général le récit classique, récit non-focalisé, ou à focalisation zéro" (2007 [1983, 1972]: 194). Más adelante Genette aclara en "Nouveau discours du récit": "[...] il me semble que le récit classique place parfois son "foyer en un point si indéterminé, ou si lointain, à champ si panoramique (le fameux 'point de vue de Dieux', ou de Sirius, dont on se demande périodiquement s'il est bien un point de vue) qu'il ne peut coïncider avec aucun personnage, et que le terme de non-focalisation, ou focalisation zéro, lui convient plutôt mieux" (2007 [1983]: 348).

${ }_{15}$ Para esta noción ver Werner Wolf: "Twentieth-century fiction is characterized by a far-reaching transformation of traditional realism. This is also true for one of the features usually combined with realism, although not confined to it: aesthetic illusion" (1990: 284).
} 
As an indifference cherished, or left to atrophy, becomes an emptiness, to this extent he had learned to become empty of Nicole, serving her against his will with negotiations and emotional neglect. One writes of scars healed, a loose parallel to the pathology of the skin, but there is no such thing in the life of an individual. There are open wounds, shrunk sometimes to the size of a pin-prick, but wounds still. The marks of suffering are more comparable to the loss of a finger, or the sight of an eye. We may not miss them, either, for one minute in a year, but if we should there is nothing to be done about it (1953: 181).

El episodio que se está narrando se detiene para pasar a la descripción de lo que ocurre en el plano psicológico de Dick y culminar con una afirmación, casi una sentencia, sobre la experiencia del dolor emocional. Las digresiones de orden reflexivo son frecuentes en esta novela - probablemente mucho más que en The Great Gatsby- y buscan un efecto de contundencia que acompaña la descripción del episodio que se esté narrando. Esta intervención de ciertas digresiones y reflexiones durante el desarrollo del relato ${ }^{16}$ es un procedimiento que Piglia utiliza igualmente en Blanco nocturno, como veremos más adelante.

En Fitzgerald esta característica ha sido identificada como una hibridación entre una escritura lírica y otra dramática —en el sentido de la narración de una acción-. De nuevo en palabras de Curnutt:

Yet Stern is correct to note that Fitzgerald's lyrical gifts and his dramatic skills could work at cross purposes. The friction is apparent in descriptive passages as well as in his preference for an omniscient point of view, which allowed him to comment on his unfolding plots (2007: 107).

La preferencia por el tipo de narrador extradiegético, si bien limita la focalización, le permite a Fitzgerald hacer excursos reflexivos e incluso líricos. La reunión de estos dos registros no es una falta de control dentro de la narración, ni una tensión que desequilibre la supuesta unicidad del hilo narrativo. Al contrario, el encuentro de lo lírico y lo dramático, y de lo narrativo y lo reflexivo dentro de una misma voz narradora contribuyen a la creación de esa atmósfera y ese tono que marcan novelas como Tender is the Night. De forma similar, Piglia opta por un narrador igualmente impersonal pero que le permite mantener su discurso reflexivo dentro del desarrollo de los episodios, sin que se tenga la impresión de una falta de continuidad de un registro al otro:

Cuando llegó al hotel, Renzi descubrió en el bar a las hermanas Belladona sentadas en una mesa. Se paró frente a la barra y pidió una cerveza. Las miró por el espejo entre el reflejo de las botellas, Ada hablaba con entusiasmo, Sofía

\footnotetext{
${ }^{16} \mathrm{El}$ caso del narrador de The Great Gatsby es distinto ya que, como se sabe, es un personaje que,
} si bien se mantiene relativamente al margen de los hechos narrados, hace parte de la historia. 
asentía, mucha intensidad entre ellas, demasiada... If it was a man. Como siempre que estaba en problemas, Renzi se acordó de un libro que había leído. La frase venía de un cuento de Hemingway, The Sea Change, que había traducido para el suplemento cultural de un diario. La literatura no cambia, siempre se puede encontrar lo que se espera, en cambio la vida... Pero ¿qué era la vida? Dos hermanas en el bar de un hotel de provincia (2010: 268).

Nuestra hipótesis es que en este caso no se trata de un discurso indirecto libre; no es la voz de Renzi la que interfiere con la del narrador extradiegético, sino que es siempre la misma voz del narrador la que hace excursos de este orden, integrando la reflexión sin que esto implique una ruptura en la narración.

\section{De Respiración artificial a Blanco nocturno: hacia el predominio de la narratividad}

A pesar de esta presencia de lo reflexivo, y con respecto a Respiración artificial o La ciudad ausente, habría que decir que la aparición de estas digresiones es cada vez menor en el narrador de Blanco nocturno, como si Piglia estuviese intentando alejarse cada vez más de ese tipo de narración explícitamente reflexiva, para volver a un narrador extradiegético tradicional y limitar el registro reflexivo a sus personajes o a elementos que se sitúan dentro de la historia misma; es, en efecto, como si su búsqueda actual estuviera volviendo a una forma narrativa más tradicional, manteniendo de vez en cuando la misma licencia que se toma el narrador de Fitzgerald en Tender is the Night: una alternancia entre los episodios narrados y una prosa reflexiva y sugestiva, ya sea respecto a la idea contemporánea de la literatura o con relación a otros temas de la historia o de la experiencia.

Consideramos que la potencia de las novelas de Piglia reside, en parte, en la hibridación de estos elementos reflexivos, que se pueden vincular con de la obra de Macedonio, con las formas narrativas que asociamos a Fitzgerald como alternancia entre lo episódico, lo lírico y lo digresivo. Sin embargo, en la fase más reciente de la producción de Piglia la idea vanguardista y macedoniana del artificio literario autónomo se incorpora solo como tema de la ficción y, por otra parte, se integra la forma narrativa secuencial y la alternancia de lo reflexivo y lo narrativo, como parte de la búsqueda de un estilo sugestivo. Todo esto sin que lo metaficcional tome un lugar privilegiado frente al hecho de contar historias. La reflexión sobre la idea de la literatura, si bien sigue infiltrándose en la construcción de las novelas del argentino, ha dejado su lugar como eje estructural y formal de la narración - como era el caso en La ciudad ausente-, para cederle el paso a una narratividad secuencial y a un lugar más clásico de lo reflexivo dentro de la narración. De esta forma, tal vez sea más productivo entender la más reciente novela de Piglia en función de la recuperación de elementos estéticos de la primera mitad del siglo XX que a partir del discurso de una estética posmoderna de la fragmentación: pero estos elementos estéticos no tienen que ver tanto con la autonomía vanguardista de Macedonio — que si bien está 
presente se ha ido diluyendo como un tema relativamente marginal dentro de las novelassino sobre todo con un particular uso de un narrador tradicional. En novelas como Plata quemada o Blanco nocturno habría que afirmar entonces el predominio del regreso a un tipo de narrador tradicional que, aun cuando es usado de una manera sutilmente reflexiva - es decir de forma similar a la de Fitzgerald - tal vez no logre la misma potencia ni el carácter sugestivo de Respiración artificial o La ciudad ausente, donde la tensión entre lo narrativo y lo reflexivo impide que la secuencialidad de la historia y la verosimilitud tradicionales se impongan como ejes de construcción ficcional. Ahora bien, a través de la obra de Piglia y más allá del agotamiento de lo que se identifica como posmoderno queda abierta la pregunta por el futuro de este regreso de la narratividad tradicional dentro de la novela en español; la cuestión de hasta qué punto escribir novelas tradicionales puede seguir considerándose experimental. 


\section{Bibliografía citada}

Bratosevich, Nicolás (1997). Ricardo Piglia y la cultura de la contravención. Buenos Aires: Atuel.

Berg, Edgardo (2003). Ricardo Piglia: un narrador de historias clandestinas. Mar del Plata: E. Balder, Universidad Nacional de Mar del Plata.

Carrión, Jorge (ed.) (2008). El lugar de Pighia. Barcelona: Candaya.

Curnutt, Kirk (2007). The Cambridge Introduction to F. Scott Fitzgerald. Cambridge: Cambridge University Press. DOI: 10.1017/CBO9780511611032

Corral, Rose (ed.) (2007). Entre ficción y reflexión, Juan José Saer y Ricardo Piglia. México, D. F.: El Colegio de México, Centro de Estudios Linguísticos y Literarios.

Bruccoli, Matthew J. y George Parker Anderson (2003). F. Scott Fitzgerald's Tender is the Night: A Documentary Volume. Dictionary of Literary Biography, Volume 273. Detroit: Gale group.

Fernández, Macedonio (1996). Museo de la Novela de la Eterna. Edición crítica, Ana María Camblong y Adolfo de Obieta, (coord). Madrid: ALLCA XX.

Fitzgerald, Francis Scott (1953 [1951]). Tender is the Night, A romance. With the Author's Final Revisions. Macolm Cowley (Ed.). New York: Charles Scribner's Sons.

Fitzgerald, Francis Scott (2005). My Lost City: Personal Essays, 1920-1940. The Cambridge Edition of the Works of F. Scott Fitzgerald. Cambridge: Cambridge University Press.

Fornet, Jorge (2005). El escritor y la tradición. La Habana: Editorial Letras Cubanas.

Garabano, Sandra (2003). Reescribiendo la nación, La narrativa de Ricardo Piglia. Ciudad Juárez: Universidad Autónoma de Ciudad Juárez.

Genette, Gérard (2007 [1983, 1972]). Discours du récit. Paris: Éditions du Seuil.

Hallward, Peter (2001). Absolutely Postcolonial: Writing Between the Singular and the Specific. Angelikai Humanities. Manchester: Manchester University Press.

Hutcheon, Linda (1988). A poetics of Postmodernism. London: Routledge. DOI: $10.4324 / 9780203358856$

Lyotard, Jean-François (1979). La condition postmoderne: rapport sur le savoir. Paris: Éditions de Minuit.

Nicol, Bran (2009). The Cambridge Introduction to Postmodern Fiction. Cambridge: Cambridge University Press. DOI: 10.1017/CBO9780511816949

Niederhoff, Burkhard (2009)."Focalization". Peter Hühn, John Pier, Wolf Schmid y Jörg Schönert (eds.) Handbook of Narratology. Berlin: W. de Gruyter: 115-123. 
Pereira, Maria Antonieta (2001). Ricardo Piglia y sus precursores. Buenos Aires: Corregidor.

Piglia, Ricardo (2000). Formas breves. Barcelona: Editorial Anagrama.

Piglia, Ricardo (2001). Crítica y ficción. Barcelona: Editorial Anagrama.

Piglia, Ricardo (2010). Blanco nocturno. Barcelona: Editorial Anagrama.

Rodríguez Pérsico, Adriana (2000 [1990]). "Introducción a Ricardo Piglia”. Jorge Fornet (ed.) Ricardo Piglia. Bogotá: Instituto Caro y Cuervo: 45-63.

Rodríguez Pérsico, Adriana (2004). Ricardo Piglia: una poética sin límites. Pittsburg: Instituto Internacional de Literatura Iberoamericana.

Stern, Milton R. (1986). "The Text Itself". Critical Essays on F. Scott Fitzgerald's Tender is the Night. Boston: G. K. Hall \& Co: 21-31.

Waugh, Patricia (1984). Metafiction, The Theory and Practice of Self-Conscious Fiction. London: Routledge.

Wolf, Werner (1990). "Illusion and Breaking Illusion in Twentieth-Century Fiction". Frederick Burwick and Walter Pape (eds.) Aesthetic Illusion, Theoretical and Historical Approaches. Berlin: De Gruyter: 284-297. 